\title{
Healthcare Associated Urinary Tract Infections in
}

\section{Medicine Wards}

\author{
Dr. Mahendra M. Sawarkar" ${ }^{* 1}$, Dr. Shushrut Mahendra Sawarkar ${ }^{2}$ \\ ${ }^{1}$ Associate Professor Dept. of Medicine CCMM Medical College Durg, CG \\ ${ }^{2}$ SR NKP Salve institute of Medical sciences, Higna Nagpur
}

Address for Correspondence

Dr. Mahendra M. Sawarkar

Associate Professor Dept. of Medicine, CCMM Medical College Durg, CG

\begin{abstract}
Health care associated infections (HCAIs) or hospital acquired infections (HAIs) are infections that occur during hospitalization but they are neither present nor incubating before hospital admission. Rapid advancement in medical technology, injudicious use of antibiotics and better adaptation of organisms to the hospital environment contribute to increase in HAIs. Healthcare-associated urinary tract infections (HAUTIs) are frequently increasing and largely preventable infections affecting the provision of healthcare. Material and Methods: Cases included patients admitted in wards of medicine department. Inclusion criteria: included patients are who were admitted for at least for past 48 hours, or readmitted in less than 14 days after their discharge from hospital. Confirmed HAI was defined as clinical signs and microbiological confirmation; potential HAI: association of several clinical signs with no microbiological confirmation; less potential HAI: a single clinical sign with no microbiological confirmation; no potential HAI: no clinical signs. Sociodemographic data from all the included patients was confirmed from medical records. All clinical history was obtained from the patients. Signs of HAI were observed and recorded. Results: A total of 528 patients were included in the studies who were admitted in the medicine wards. Of the 528 patients $276(52.3 \%)$ were male and $252(47.7 \%)$ were female. 87 patients were diagnosed as HAI patients of which $37(42.5 \%)$ were male and $50(57.5 \%)$ were female. Mean age of HAI patients was $51.3 \pm 15.74$ while total mean of age was $44.2 \pm 24.77$. Mean age of male with HAI $49.5 \pm 11.99$ and female was $52.4 \pm 13.47$. Of the total 10 culture positive patients with HAI Escherichia coli was isolated in 3 cases, Klebsiella pneumonia, Pseudomonas aeruginosa and Staphylococcus aureus were isolated in 2 cases each. While Acinetobacter spp. was isolated in 1 case. Conclusion: This study shows the prevalence of HAI in Urinary tract infection as $15.3 \%$ which was quite high but there is no mortality due to UTI and HAI. Periodic active surveillance over a longer period is required to evaluate the efficacy of preventive measures.
\end{abstract}

\section{Introduction}

Health care-associated infections (HAIs) burden patients, can complicate treatments, prolong the hospital stay of patients, increase treatment costs, and can be life-threatening. The Centers for Disease Control and Prevention (CDC) report recommends attempting to prevent these infections through appropriate antibiotic use and infection prevention practices. ${ }^{[1]}$ Health care associated infections (HCAIs) or hospital acquired infections (HAIs) are infections that occur during hospitalization but they are neither present nor incubating before hospital admission. Rapid advancement in medical technology, injudicious use of antibiotics and better adaptation of organisms to the hospital environment contribute to increase in HAIs. ${ }^{[2]}$ According to WHO in 2015, over 1.4 million people across the globe were affected by HAI. ${ }^{[3]}$ Approximately about half all cases of HCAIs are associated with medical devices and implants. ${ }^{[4]}$ Healthcare-associated urinary tract infections (HAUTIs) are frequently increasing and largely preventable infections affecting the provision of healthcare. The point prevalence of HAUTIs is around $1.4 \%$, with HAUTIs responsible for about $17 \%$ to $36 \%$ of all HCAIs, ${ }^{[5,6]}$ and Catheterassociated urinary tract infection (CAUTI) accounts for up to $80 \%$ of these. ${ }^{[7]}$ In most of the cases there is improper insertion of the catheter and CAUTI risk increases considerably with duration of catheterisation. $^{[8]}$ In HAUTI majority of causative agents are Gram-negative bacteria and emergence of antimicrobial resistance is of particular concern with respect to urinary tract infections (UTIs). ${ }^{[9]}$ In a European multicentre study posted that the proportion of infected patients in intensive care units can be as high as $51 \%$; most of these are health care associated. ${ }^{[10]}$

\section{Material and Methods}

Present study was carried out in department of Medicine at CCM Medical College and Hospital. In this study population included patients admitted in wards of medicine department. Inclusion criteria: included patients are who were admitted for at least for past 48 hours, or readmitted in less than 14 days after their discharge from hospital. Informed written consent was obtained from all the patients. Exclusion criteria: Patients who completed less than 48 hours of admission and those who received only outpatient care or had exclusively day hospital care were not included.

Healthcare-associated infection (HAI) was defined as infections seen after 48 hours admission or within 14 days following 


\section{International Journal of Innovative Research in Medical Science (IJIRMS) Volume 03 Issue 12 Dec 2018, ISSN: 2455-8737, Imp. Factor - 4.102 Available online at - $\underline{w w w . i j i r m s . i n}$}

discharge from the hospital. Some terms related to HAI have been used as part of this study: Confirmed HAI was defined as clinical signs and microbiological confirmation; potential HAI: association of several clinical signs with no microbiological confirmation; less potential HAI: a single clinical sign with no microbiological confirmation; no potential HAI: no clinical signs.

Bacteremia was defined as presence of bacteria in the vascular system confirmed through at least one positive blood culture. However, blood culture must be confirmed by clinical signs such as fever (Temperature $\geq 38.5^{\circ} \mathrm{C}$ ) or hypothermia (Temperature $\leq$ $\left.36.5^{\circ} \mathrm{C}\right)$, chills or hypotension.

Sociodemographic data from all the included patients was confirmed from medical records. All clinical history was obtained from the patients. Signs of HAI were observed and recorded these signs were: hyperthermia Temperature $>38^{\circ} \mathrm{C}$; Hypothermia Temperature $\leq 36^{\circ} \mathrm{C}$; chills; urinary tract signs or lumbar pain, suprapubic, dysuria, IC-urgency, or burning micturition. HIV status of all the patients, antibiotics administered during admission, site of HAI, existence of invasive device like urinary catheter, venous catheter, sensitivity to antibiotics, and evolution of healthcare associated infection was done.

Data entry was done in Microsoft Excel 2010 edition. All data analysis was carried out through SPSS software, Continuous variables were expressed in form of mean values with their standard deviation, or medians with their interquartile ranges. Categorical variables were expressed in percentage.

\section{Results and Observations}

A total of 528 patients were included in the study who were admitted in the medicine wards. Age group of patients was 28 to 67 years. Out of 825 patients included in the study, 87 (16.5\%) presented one or several signs suggestive of HAI and they received microbiological tests. $10(11.49 \%)$ cases were confirmed or potential HAI.

Table 1: Sociodemographic data

\begin{tabular}{|l|c|c|c|}
\hline Variable & Total & Male (\%) & Female (\%) \\
\hline Total patients & 528 & $276(52.3 \%)$ & $252(47.7 \%)$ \\
\hline HAI patients & 87 & $37(42.5 \%)$ & $50(57.5 \%)$ \\
\hline $\begin{array}{l}\text { Mean Age } \\
\text { (Total Patients) }\end{array}$ & $44.2 \pm 24.77$ & $47.6 \pm 21.51$ & $43.3 \pm 19.55$ \\
\hline $\begin{array}{l}\text { Mean Age } \\
\text { (HAI patients) }\end{array}$ & $51.3 \pm 15.74$ & $49.5 \pm 11.99$ & $52.4 \pm 13.47$ \\
\hline
\end{tabular}

Of the 528 patients $276(52.3 \%)$ were male and $252(47.7 \%)$ were female. 87 patients were diagnosed as HAI patients of which 37 $(42.5 \%)$ were male and $50(57.5 \%)$ were female. Mean age of HAI patients was $51.3 \pm 15.74$ while total mean of age was $44.2 \pm$ 24.77. Mean age of male with HAI $49.5 \pm 11.99$ and female was $52.4 \pm 13.47$.

Table 2 Healthcare associated infection and urinary catheter

\begin{tabular}{|c|c|c|c|}
\hline & \multicolumn{3}{|c|}{$\begin{array}{c}\text { Health care associated } \\
\text { infection }(\mathrm{n}=528)\end{array}$} \\
\hline Urinary Cather & Yes & No & Total \\
\hline Yes & $81(15.3 \%)$ & $413(78.2 \%)$ & $494(93.6 \%)$ \\
\hline No & $6(1.1 \%)$ & $28(5.3 \%)$ & $34(6.4 \%)$ \\
\hline Total & $87(16.5 \%)$ & $441(83.5 \%)$ & $528(100 \%)$ \\
\hline
\end{tabular}

Of the total 87 HAI cases $81(15.3 \%)$ had urinary catheter while 6 $(1.1 \%)$ dosent had catheter over all prevalence of HAI was 87 (16.5\%). Of the total $494(93.6 \%)$ patients who had urinary catheter 413 (78.2\%) doesn't show HAI. While 34 (6.4\%) who doesn't had catheter $28(5.3 \%)$ had no HAI.

Table 3: Microbiological profile

\begin{tabular}{|l|c|c|}
\hline Organism & $\mathrm{N}=10$ & $\%$ \\
\hline Escherichia coli & 3 & $30 \%$ \\
\hline Klebsiella pneumonia & 2 & $20 \%$ \\
\hline Pseudomonas aeruginosa & 2 & $20 \%$ \\
\hline Staphylococcus aureus & 2 & $20 \%$ \\
\hline Acinetobacter spp. & 1 & $10 \%$ \\
\hline
\end{tabular}

Of the total 10 culture positive patients with HAI Escherichia coli was isolated in 3 cases, Klebsiella pneumonia, Pseudomonas aeruginosa and Staphylococcus aureus were isolated in 2 cases each. While Acinetobacter spp. was isolated in 1 case.

\section{Discussion and Conclusion}

Health-care-associated infection is in most of the settings determines clinical outcome among patients admitted in critical care areas. Device-associated infections has become an integral feature of infection control in most of the hospitals. HAI includes catheter-associated urinary tract infections (CAUTI), central-lineassociated blood stream infections (CLABSI), and ventilatorassociated pneumonias (VAP). ${ }^{[11]}$ The hospitals in developed countries generate their infection-control surveillance data from time to time as this is important for empirically treating infections, especially in the intensive care unit (ICU) setting. ${ }^{[12]}$ HAI is a cause of increased morbidity, mortality and resource expenditure in different healthcare settings. Incidence of HAI has been reported in different hospitals from various parts of the world. More than $20 \%$ of patients in Intensive Care Unit (ICU) may be infected with various HCAI with mortality rate of $>30 \%{ }^{[13]}$ During hospitalization, various HCAI directly related to different invasive procedures such as urinary tract infection, pneumonia, blood stream infection, surgical site infection are often encountered. ${ }^{[14]}$ Surveillance of HAI is an essential element so as to know the current prevalence of the condition and to identify potential risk factors.

In our study prevalence of HAI urinary tract infection was 81 (15.3\%).In a study by Agrawal R et al. ${ }^{[15]}$ HAI was $6.67 \%$ they observed the device utilization ratio of urinary catheters was highest $(0.20)$ and Urinary tract infection was the most common $(71.4 \%)$. In a study by Laborde $\mathrm{G}$ et al., reported the infection rate to be $36.3 \%$ from 314 patients treated longer than 48 hours in neurosurgical ICU. ${ }^{[16]}$ Prevalence of HAI in a study by DIAet al in Senegal $10.9 \%$. $^{[17]}$

CDC (centre for disease surveillance Centre USA), in collaboration with other organizations, has developed guidelines for the prevention of Catheter-associated UTIs and other types of healthcare-associated infections ${ }^{[18]}$ in which they recommended: appropriate urinary catheter use, Proper techniques for urinary catheter insertion, Proper techniques for urinary catheter maintenance, Quality improvement programs and surveillance. 


\section{International Journal of Innovative Research in Medical Science (IJIRMS) Volume 03 Issue 12 Dec 2018, ISSN: 2455-8737, Imp. Factor - 4.102 Available online at - www. ijirms.in}

In our study out of $494(93.6 \%)$ with urinary catheter 81 (15.3\%) had HAI. While $6(1.1 \%)$ had HAI but no urinary catheter. Bacteria have been part of the normal human microbiota for eons and usually do not cause signs or symptoms of infection but may be responsible for the HAI. ${ }^{[19]}$ Hospitalized patients are at high risk of colonization with health care-associated pathogens. in a survey of 143 Canadian hospitals in 2012 it was found that among their hospitalized patients, $4.5 \%$ were colonized or infected with MRSA, $2.7 \%$ were colonized or infected with vancomycin-resistant enterococci (VRE), $1.4 \%$ were colonized or infected with $\mathrm{C}$. difficile, $1.3 \%$ were colonized or infected with an extendedspectrum $\beta$-lactamase (ESBL)-producing organism, and $0.1 \%$ were colonized or infected with carbapenem-resistant Enterobacteriaceae (CRE) ${ }^{[20]}$ In a study by Modi et al with indwelling devices found that of the $15 \%$ who were colonized with multidrug-resistant Acinetobacter baumannii, nearly half of those colonisations recurred over time. ${ }^{[21]}$

In this study cin culture confirmed HAI cases Escherichia coli was isolated in 3 cases, Klebsiella pneumonia, Pseudomonas aeruginosa and Staphylococcus aureus were isolated in 2 cases each. While Acinetobacter spp. was isolated in 1 case. Similar results were shown by Agrawal et al in their study in which common organisms responsible for HAI were Klebsiellapneumoniae followed by Escherichia coli in Catheter Associated Urinary Tract Infection. ${ }^{[15]}$

In our study no mortality was observed in HAI group. Similar wit the study by Agrawal et al. ${ }^{[15]}$

This study shows the prevalence of HAI in Urinary tract infection as $15.3 \%$ which was quite high but the positive perspective is there is no mortality due to UTI and HAI. All guidelines were followed for the insertion and maintenance off the catheter. But to evaluate the efficacy of preventive measures, periodic active surveillance over a longer period is required.

\section{References}

[1] Centers for Disease Control and Prevention. Antibiotic resistance threats in the United States, 2013. http://www.cdc.gov/drugresistance/threat-report2013/pdf/ar-threats-2013-508.pdf

[2] Deorukhkar S. C., Saini S., Mathew S. Non-albicans Candida infection: an emerging threat. Interdisciplinary Perspectives on Infectious Diseases. 2014; 2014:7.

[3] WHO (2015) Clean Care Is Safer Care Why a Global Challenge on Healthcare-Associated Infections. Who Media Center. 1-2. http://www.who.int/gpsc/background/fr/\#

[4] Kojic E. M., Darouiche R. O. Candida infections of medical devices. Clinical Microbiology Reviews. 2004;17(2):255-267. doi: 10.1128/cmr.17.2.255267.2004.

[5] Zarb, P., Coignard, B., Griskeviciene, J. et al. The European Centre for Disease Prevention and Control (ECDC) pilot point prevalence survey of healthcareassociated infections andantimicrobial use. Euro Surveill. 2012; 17

[6] Gardner, A., Mitchell, B.G., Beckingham, W., and Fasugba, O. A point prevalence cross-sectional study of healthcare-associated urinary tract infections in six Australian hospitals. BMJ Open. 2014; 4

[7] Parker V, Giles M, Graham L, et al. Avoiding inappropriate urinary catheter use and catheter-associated urinary tract infection (CAUTI): a pre-post control intervention study. BMC Health Serv Res. 2017;17 (1):314. Published 2017 May 2. doi:10.1186/s12913$017-2268-2$

[8] Gould CV, Umscheid CA, Agarwal RK, Kuntz G, Pegues DA. Healthcare Infection Control Practices Advisory Committee: Guideline for prevention of catheter-associated urinary tract infections 2009. Infect Control HospEpidemiol. 2010;31(4):319-326.

[9] Chen, L.F., Chiu, C.-T., Lo, J.-Y. et al. Clinical characteristics and antimicrobial susceptibility pattern of hospitalised patients with community-acquired urinary tract infections at a regional hospital in Taiwan. Healthcare Infect. 2014; 19: 20-25

[10] World Health Organization. Health care-associated infections fact sheet. Available at: http://www.who.int/gpsc/country_work/gpsc_ccisc_fact_ sheet_en.pdf, 2016

[11] Horan TC, Andrus M, Dudeck MA. CDC/NHSN surveillance definition of health care-associated infection and criteria for specific types of infections in the acute care setting.Am J Infect Control. 2008 Jun; 36(5):309-32.

[12] Haley RW, Culver DH, White JW, Morgan WM, Emori TG, Munn VP, Hooton TM. The efficacy of infection surveillance and control programs in preventing nosocomial infections in US hospitals..Am J Epidemiol. $1985 \mathrm{Feb} ; 121(2): 182-205$.

[13] Orsi GB, Scorzolini L, Franchi C, Mondillo V, Rosa G, Venditti M. Hospital-acquired infection surveillance in a neurosurgical intensive care unit.J Hosp Infect. 2006 Sep; 64(1):23-9.

[14] Wałaszek M. The analysis of the occurrence of nosocomial infections in the neurosurgical ward in the District Hospital from 2003-2012.PrzeglEpidemiol. 2015; 69(3):507-14, 619-23.

[15] Agarwal R, Mohapatra S, Rath GP, Kapil A. Active Surveillance of Health Care Associated Infections in Neurosurgical Patients. J ClinDiagn Res. 2017;11(7):DC01-DC04.

[16] Laborde G, Grosskopf U, Schmieder K, Harders A, Klimek L, Hardenack M, Gilsbach JM Nosocomial infections in a neurosurgical intensive care unit. Anaesthesist. 1993 Oct; 42(10):724-31.

[17] Dia, N.M., Ka, R., Dieng, C., Diagne, M.L., Fortes, L., et al. (2008) Results of the Prevalence Survey of Healthcare-Associated Infections in the Fann CHNU (Dakar, Senegal). Medicine and Infectious Diseases, 38, 270-274.

[18] https://www.cdc.gov/hai/ca_uti/uti.html

[19] Lebreton F, van Schaik W, McGuire AM, Godfrey P, Griggs A, Mazumdar V, Corander J, Cheng L, Saif S, Young S, Zeng Q, Wortman J, Birren B, Willems RJ, Earl AM, Gilmore MSEmergence of epidemic multidrug-resistant Enterococcus faecium from animal and commensal strains.MBio. 2013 Aug 20; 4(4).

[20] Williams V, Simor AE, Kiss A, McGeer A, Hirji Z, Larios OE, Moore C, Weiss K, Infection Prevention and 
Control-Canada.Is the prevalence of antibiotic-resistant organisms changing in Canadian hospitals? Comparison of point-prevalence survey results in 2010 and 2012.Clin Microbiol Infect. 2015 Jun; 21(6):553-9.

[21] Mody L, Gibson KE, Horcher A, Prenovost K, McNamara SE, Foxman B, Kaye KS, Bradley S.
Prevalence of and risk factors for multidrug-resistant Acinetobacter baumannii colonization among high-risk nursing home residents.Infect Control HospEpidemiol. 2015 Oct; 36(10):1155-62. 